\title{
BMJ Open Kids' Outcomes And Long-term Abilities (KOALA): protocol for a prospective, longitudinal cohort study of mild traumatic brain injury in children 6 months to 6 years of age
}

\author{
Miriam H Beauchamp (D) , ${ }^{1,2}$ Fanny Dégeilh, ${ }^{1,2,3}$ Keith Yeates (D) ,,5,6 \\ Isabelle Gagnon, ${ }^{7,8}$ Ken Tang, ${ }^{9}$ Jocelyn Gravel, ${ }^{10}$ Antonia Stang, ${ }^{11,12}$ \\ Brett Burstein, ${ }^{13}$ Annie Bernier, ${ }^{14}$ Catherine Lebel, ${ }^{5,6,15}$ Ramy El Jalbout, ${ }^{16}$ \\ Sonia Lupien, ${ }^{17}$ Louis de Beaumont, ${ }^{18}$ Roger Zemek (D) , ${ }^{19}$ Mathieu Dehaes, ${ }^{1}$ \\ Sylvain Deschênes, ${ }^{16}$ On behalf of the PERC KOALA Project
}

To cite: Beauchamp MH, Dégeilh F, Yeates $\mathrm{K}$, et al. Kids' Outcomes And Long-term Abilities (KOALA): protocol for a prospective, longitudinal cohort study of mild traumatic brain injury in children 6 months to 6 years of age. BMJ Open 2020;10:e040603. doi:10.1136/ bmjopen-2020-040603

- Prepublication history for this paper is available online. To view these files, please visit the journal online (http://dx.doi. org/10.1136/bmjopen-2020040603).

Received 19 May 2020 Revised 05 August 2020 Accepted 26 August 2020

D) Check for updates

(c) Author(s) (or their employer(s)) 2020. Re-use permitted under CC BY-NC. No commercial re-use. See rights and permissions. Published by BMJ.

For numbered affiliations see end of article.

\section{Correspondence to}

Dr Miriam H Beauchamp; miriam.beauchamp@umontreal. ca

\section{ABSTRACT}

Introduction Mild traumatic brain injury (mTBI) is highly prevalent, especially in children under 6 years. However, little research focuses on the consequences of $\mathrm{mTBI}$ early in development. The objective of the Kids' Outcomes And Long-term Abilities (KOALA) study is to document the impact of early mTBI on children's motor, cognitive, social and behavioural functioning, as well as on quality of life, stress, sleep and brain integrity.

Methods and analyses KOALA is a prospective, multicentre, longitudinal cohort study of children aged 6 months to 6 years at the time of injury/recruitment. Children who sustain mTBI $(n=150)$ or an orthopaedic injury $(n=75)$ will be recruited from three paediatric emergency departments (PEDs), and compared with typically developing children (community controls, $n=75$ ). A comprehensive battery of prognostic and outcome measures will be collected in the PED, at 10 days, 1,3 and 12 months postinjury. Biological measures, including measures of brain structure and function (magnetic resonance imaging, MRI), stress (hair cortisol), sleep (actigraphy) and genetics (saliva), will complement direct testing of function using developmental and neuropsychological measures and parent questionnaires. Group comparisons and predictive models will test the a priori hypotheses that, compared with children from the community or with orthopaedic injuries, children with $\mathrm{mTBI}$ will (1) display more postconcussive symptoms and exhibit poorer motor, cognitive, social and behavioural functioning; (2) show evidence of altered brain structure and function, poorer sleep and higher levels of stress hormones. A combination of child, injury, socioenvironmental and psychobiological factors are expected to predict behaviour and quality of life at 1, 3 and 12 months postinjury.

Ethics and dissemination The KOALA study is approved by the Sainte-Justine University Hospital, McGill University Health Centre and University of Calgary Conjoint Health Research Ethics Boards. Parents of participants will provide written consent. Dissemination will occur through
Strengths and limitations of this study

- The Kids' Outcomes And Long-term Abilities (KOALA) study will be the first large, prospective, multicentre cohort study to focus specifically on mild traumatic brain injury (mTBI) in early childhood.

- The study will be the first to include objective measures of parent and child stress after mTBI.

- The KOALA study will systematically document brain integrity and sleep in young children using state-ofthe-art, developmentally appropriate methods.

- There is currently no consensus on diagnostic criteria for identifying $\mathrm{mTBI}$ in children 5 years and under; the definition used here, therefore, relies on previous empirical work in this age group.

- The study includes only children recruited in urban paediatric emergency departments, and therefore, the sample may not be representative of children who sustain early $\mathrm{mTBI}$, but are not evaluated in emergency departments.

peer-reviewed journals and an integrated knowledge translation plan.

\section{INTRODUCTION}

Paediatric mild traumatic brain injury (mTBI, also called concussion) is a highly prevalent, chronic and complex health problem. Children under 6 years of age constitute a highrisk group, with a yearly rate of TBI of 1.85 per 100 children (compared with $<1.17$ in older children), ${ }^{1-3}$ and children under the age of 3 years have an especially high incidence of visits to the emergency department for head injury. ${ }^{4}$ International organisations have made a strong case for the urgency of large-scale collaborative studies on the 
prevention, diagnosis and treatment of paediatric TBI, ${ }^{56}$ yet few multicentre studies focus on mTBI sustained in early childhood (where "early" is defined here as occurring in infants, toddlers and preschoolers before 6 years of age).

Young age at injury influences morbidity due to the unique pathophysiology associated with insults to the immature brain. ${ }^{7}$ Given the same mechanical force, young brains are more likely to suffer severe injury because of the relatively large and heavy heads of small children and their weak cervical ligaments and muscles, as well as the fragility of their thin, pliable skulls and unfused cerebral sutures. ${ }^{8}$ TBI may also be especially debilitating early in development due to the vulnerability of the maturing brain and instability of emerging functional capacities. For instance, appropriate cognitive and social development are critically dependent on the integrity of particular brain structures at early stages of development and on children attaining key developmental milestones. ${ }^{9} 10$

Most studies of early TBI focus on moderate to severe injuries and highlight heightened levels of externalising behaviour problems, as well as lower levels of attentional, language, executive, social, academic and intellectual functioning, with problems persisting in some cases into adolescence and early adulthood. ${ }^{11-16}$ Evidence suggests worse outcomes in preschool children with TBI compared with those aged 6 and older, ${ }^{17-21}$ and in infants compared with preschoolers in some areas of functioning. ${ }^{22}$ One of the largest single-centre, prospective cohorts of early TBI (Learning to Interact Optimises Neurons (LION) Project: $18-60$ months, all TBI severities) indicates that even mTBI sustained early in life can critically disrupt social functioning, such as affect recognition and the ability to understand other people's perspectives (theory of mind),${ }^{23-25}$ and can result in reduced quality parentchild interactions and elevated behaviour problems, ${ }^{26} 27$ in some cases altering expected developmental trajectories of social and behavioural functioning more than 18 months postinjury. ${ }^{28}$

As in older children and adults who sustain TBI, outcomes are likely to be influenced by the complex interaction of injury factors (eg, timing, severity, location), demographic and premorbid factors (eg, age at injury, temperament and character traits, resilience, preinjury functioning) and social-environmental factors (eg, socioeconomic status (SES), parent mental health, familial functioning). ${ }^{29-31}$ However, risk and protective factors have not been extensively studied after early TBI and are likely to contribute differentially when compared with older children and adults given that early childhood is subject to different influences (eg, greater reliance of young children on their parents). ${ }^{32} 33$

Studies in older children with TBI indicate that biological and psychological markers such as TBI-induced neuropathology, ${ }^{34} 35$ genetics, ${ }^{36}$ sleep ${ }^{37} 38$ and stress response may be useful prognostic markers of recovery, but they have rarely been studied after early TBI. Due to methodological limitations (eg, motion artefacts, ${ }^{39}$ such as blurring and ghosting in the image, resulting from the prolonged time needed to stay still while sufficient data is collected to form an image; use of sedation) associated with the challenge of performing brain imaging in young children, no magnetic resonance imaging (MRI) study of early TBI includes advanced research sequences (eg, diffusion MRI, susceptibility weighted imaging (SWI), resting state functional MRI). These sequences are known to have the potential to detect subtle brain changes associated with mTBI. ${ }^{40-42}$ To our knowledge, only one study has investigated the contribution of genetic factors to recovery after early TBI. ${ }^{43}$ Fatigue or sleep-wake disturbances may exacerbate problems after TBI, ${ }^{38}$ but again only one study has used an objective measure (actigraphy) to document this association after early TBI. ${ }^{44}{ }^{45}$ Finally, stress response induced by the injury in both the child and his/her family have been shown to affect outcome after childhood TBI, ${ }^{46-48}$ but these effects have only been studied with subjective stress questionnaires. ${ }^{27}$

In sum, well-controlled, prospective cohort studies that comprehensively assess prognostic features and outcomes of early mTBI are lacking. Little is known about the combined risk factors for poor outcome and patterns of vulnerability and resilience across functional domains after early mTBI.

\section{OBJECTIVES}

The aims of this multicentre, prospective, longitudinal study are therefore to:

1. Document the consequences of mTBI sustained during early childhood in terms of postconcussive symptoms (PCS), motor, cognitive, social and behavioural functioning, as well as sleep quality, stress and quality of life.

2. Assess the neuropathological consequences of early mTBI and evaluate the incremental prognostic value of advanced neuroimaging biomarkers.

3. Determine the combined contribution of host (child), injury, socialenvironmental and psychobiological factors on outcome after early mTBI.

The main study hypotheses are :

A) infants, toddlers and preschoolers (ie, children under the age of 6 years) with mTBI will display increased PCS in the first month postinjury and exhibit poorer motor, cognitive, social and behavioural functioning, as well as disrupted sleep patterns, increased stress hormone levels (child and parent) and poorer quality of life, compared with comparison children from the community or with orthopaedic injuries.

B) advanced MR neuroimaging sequences will allow detection of subtle changes in brain structure and connectivity after early mTBI; it is expected that microhaemorraghic lesions will be found in some participants and that those with mTBI will have reduced resting-state connectivity.

C) A combination of host (eg, child age, sex, genetics, premorbid temperament, resilience, adaptive skills, 
behaviour, cognition), socioenvironmental (eg, parent mental health, family functioning, parent-child interactions, SES), injury (eg, injury severity markers, PCS, neuropathology) and psychobiological (eg, stress, sleep) factors will jointly contribute to predicting global functional outcome (eg, PCS, behaviour, quality of life) at 1, 3 and 12 months postinjury.

\section{METHODOLOGY \\ Design}

This is an ongoing multisite, prospective, longitudinal cohort study of mTBI sustained in children between the ages of 6 months and 6 years. Eligible children presenting with $\mathrm{mTBI}$ to three urban paediatric emergency departments (PEDs; CHU Sainte-Justine (CHUSJ), Montreal Children's Hospital-McGill University Health Centre, Alberta Children's Hospital (ACH)) will be recruited to participate and will be followed for 12 months post-injury (start date: April 12, 2019, projected end date: September 2022).

\section{Patient and public involvement}

A patient representative (parent of toddler with mTBI) was included on the funding application that led to this project and contributed to establishing programme priorities and patient perspective. We also received input from a focus group of parents of young children with mTBI on their postinjury experience and regarding commonly observed symptoms and behaviours after mTBI. We intend to disseminate the main findings to participants' families by way of an integrated knowledge translation plan and will seek patient/public involvement in the method choice and application thereof.

\section{Participants}

Participants (6 months to 5.99 years of age at injury (mTBI/orthopaedic injury, OI) or recruitment (community controls, CC) will be recruited over a period of approximately 2 years. Children with non-intentional mTBI $(n=150)$ will be identified using early childhoodspecific criteria (described below) and compared with group-matched OI $(\mathrm{n}=75)$ and typically developing community comparison groups $(\mathrm{CC}, \mathrm{n}=75)$ at a 2:1 recruitment ratio. An OI comparison group is thought to more accurately mimic the general trauma and injury confounds of mTBI (eg, pain, traumatic accident, stress, hospital-related experience) and thus be more comparable to individuals with mTBI than CC. However, recent evidence suggests that the advantages of an OI group are not as substantial as previously thought, at least for preschool children. ${ }^{49}{ }^{50}$ Both OI and CC groups will be be included here to ensure the findings in the mTBI group are brain injury-specific, given that previous work in this age group has not verified the comparability of OI and CC groups on all of the study variables of interest. ${ }^{51}$

\section{Inclusion criteria}

Mild traumatic brain injury

MTBI will be defined as per operational criteria developed based on previous work in this age group such as by
Osmond et $a l,{ }^{52}$ in the LION cohort,${ }^{26}$ and in the Canadian paediatric mTBI Common Data Elements project. ${ }^{53}$ Thus, a child will be enrolled in the mTBI group according the the following criteria.

1. Aged 6 months to 5.99 years old.

2. Presenting to the PED within 48 hours of injury (transfer from outside hospital is not an exclusion criterion as long as it occurs within 48 hours of injury).

3. Sustained a mTBI as per the following:

- Witnessed, non-intentional, traumatic event (hit, fall, motor vehical accident, etc.) resulting in a nonpenetrating injury.

- Glasgow Coma Scale (GCS) score between 13 and 15; using an age appropriate GCS

WITH one or more of the following.

- Palpable skull fracture.

- Headache(s).

- Altered mental status (including agitation, somnolence, slow response, repetitive questioning, confusion).

- Excessive irritability (more than usual) or "not acting normally' (according to parents).

- Loss of consciousness (any).

\section{Orthopaedic injury}

Participants will be enrolled in the OI group if:

1. Aged 6 months to 5.99 years.

2. Presenting to the PED within 48 hours of injury for an upper or lower limb trauma leading to a final diagnosis of simple fracture, sprain, contusion or other trauma to an extremity.

\section{Community controls}

Children aged 6 months to 5.99 years will be recruited in Montreal and Calgary and will represent same-age peers assumed to have a typical course of neurobiological development.

\section{Exclusion criteria}

$\mathrm{mTBI} / 0 \mathrm{I}$ groups

Children will be excluded in the following cases:

1. Hypoxia, hypotension or shock during or following the injury (ie, systolic blood pressure below 1 SD for age, O2 saturation $<95 \%$ requiring a medical intervention (eg, oxygen supply), clinical signs of hypoperfusion).

2. Administration of sedative medication (propofol, ketamine, nitrous oxide, midazolam, benzodiazepines; opioid administration is not an exclusion if used for pain management).

3. Surgical intervention following the injury.

4. Injury related to suspected or known abuse or assault (non-accidental).

5. Participating in a clinical intervention trial (ie, receiving anything other than standard clinical care).

6. Legal guardian not present or child in foster care.

All groups

1. Insufficient mastery of English or French to complete questionnaires (parents) or assessment (child). 


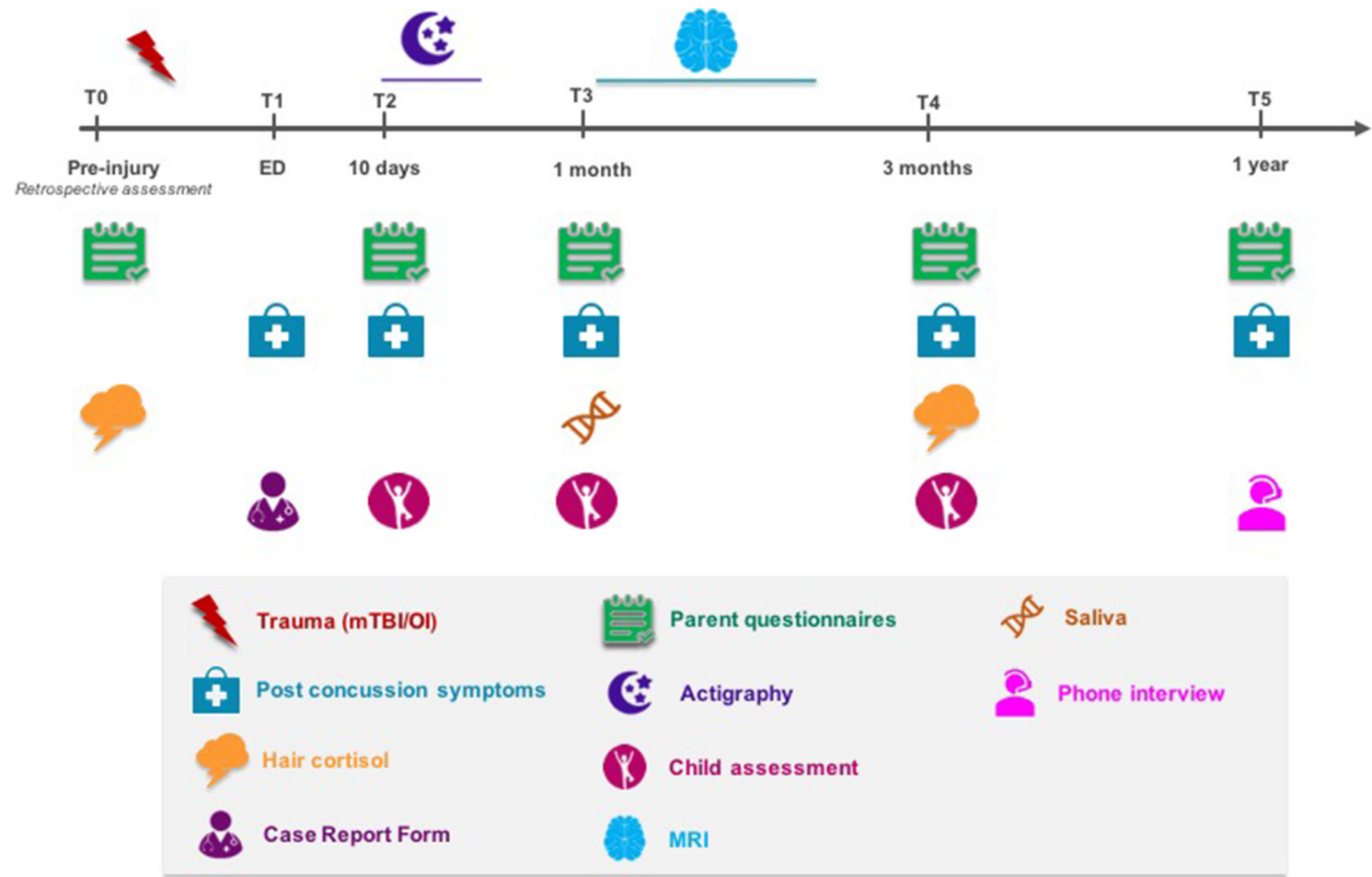

Figure 1 KOALA study summary procedure and timeline for data collection. ED, emergency department; KOALA, Kids' Outcomes And Long-term Abilities; MRI, magnetic resonance imaging, mTBI, mild traumatic brain injury; OI, orthopaedic injury.

2. Diagnosed, severe premorbid congenital, neurological, developmental, psychiatric or metabolic disorder (children who receive a diagnosis over the course of the study will be excluded from subsequent timepoints, except in the case of attention-deficit/hyperactivity disorder or learning disabilities, which are risk factors for poor outcome after TBI and frequent comorbidities. ${ }^{54-56}$

3. Children born with a history of prematurity (Gestational age $<36$ weeks).

4. History of prior TBI serious enough to warrant a visit to the PED (children who sustain a subsequent mTBI over the course of the study will be kept in the study; those in the OI and CC groups will be offered re-enrolment into the mTBI group, and those in the mTBI group will be recategorised into a 'multiple mTBI' subgroup).

5. A child or primary caregiver currently taking any of the following medications will be excluded from the cortisol analyses: neuroleptics, antidepressants, anxiolytiques, glucocorticosteroids, antiepileptics, sedatives, cholesterol medications, antiinflammatories.

\section{Procedures}

See figure 1 for a summary of the study procedure and timing of assessments and data collection.

\section{Screening and recruitment}

All children presenting to the PED and fulfilling the inclusion/exclusion criteria will be immediately identified by the study PED research personnel, who will check the PED log for presentations corresponding to
mTBI and OI criteria. Information on number of children screened will be entered into a screening log for construction of a patient flow chart, as per Strengthening the Reporting of Observational Studies in Epidemiology clinical research guidelines. ${ }^{57}$ All mTBI and OI fulfilling inclusion/exclusion criteria will be invited to participate in the study; those who agree will sign consent forms at the time of recruitment in the PED. For children whose parents decline participation, only their sex and age will be kept to determine any recruitment bias. Children in the CC group will be recruited via pamphlets and signs in local daycares, kindergartens, early childhood care centres, community centres and locations frequented by parents of young children (eg, breasfeeding clinics, play centres, baby stores).

\section{Data collection}

Acute injury stage

Day of injury will constitute the initial time point of the study $\left(\mathrm{T}_{1}\right)$ and corresponds to the acute injury stage in the PED. As soon as consent is obtained, the following $T_{1}$ measures will be obtained: the parent will complete an observational report of their child's PCS using the Report of Early Childhood Traumatic Injury Observations and Symptoms (REACTIONS ${ }^{58}$ ) and the research personnel will complete a behavioural observation measure of pain in infants and children, the Face Legs Activity Cry and Consolability (FLACC) ${ }^{59} 60$ scale. The research personel will also complete a case report form documenting acute signs and symptoms of mTBI based on medical record data (ie, loss of consciousness; GCS scores; mechanism of 
injury; neurological status; clinical interventions and treatments). During the PED visit, the research personnel will also collect a parent and child hair samples (minimum 5 $\mathrm{mg}$, up to $3 \mathrm{~cm}$ in length) for the measurement of preinjury stress hormone levels (ie, cortisol).

\section{Retrospective preinjury estimates}

An approximation of the child and parent's preinjury state will be obtained by having parents complete a series of questionnaires pertaining to functioning before the injury $\left(\mathbf{T}_{0}\right)$ in the following domains: PCS, behaviour and socioemotional skills, social cognition and competence, family functioning, parent mental health and quality of life. The parents will be invited to complete these preinjury measures as soon as possible following the injury and before the first follow-up at 10 days postinjury $\left(\mathrm{T}_{2}\right)$.

\section{Follow-up assessments}

Follow-up time points include completion of child assessments and parent questionnaires at 10 days postinjury $\left(\mathrm{T}_{2}\right)$, 1-month postinjury $\left(\mathrm{T}_{3}\right)$ and 3 months postinjury $\left(\mathrm{T}_{4}\right)$, as well as a telephone follow-up (semistructured interview) and parent questionnaires 12 months postinjury $\left(T_{5}\right)$. In order to manage the rapid timeframe between the PED $\left(\mathrm{T}_{1}\right)$ and $\mathrm{T}_{2}$ visit at 10 days postinjury, the study coordinator will contact the family within 24 hours to book the appointment and an email confirmation will be sent as well as a link for completing the preinjury questionnaires.

\section{Child}

For the child assessments, participants will be assessed by trained research assistants in a standard evaluation room. Assessments will last between 30 and $60 \mathrm{~min}$ and will consist of a combination of paper-pencil, computer and observational measurements of a range of domains: global development, language, executive functions, motor skills, social cognition and competence, socioemotional functioning, pain and parent-child interactions (table 1). Children will also complete a sleep quality assessment (actigraphy), saliva sample collection for genetic markers assessment (optional) and hair sample collection for stress measurement (when possible). Children 24 months and older will also answer some simple, qualitative questions about the accident related to their injury (Injury Story). All assessments will be videotaped for future scoring, coding and reference. Children 36 months and older will additionally complete an MRI examination.

\section{Parent}

At each timepoint, the child's primary caregiver will complete questionnaires measuring a range of domains: Demographics and medical history (including injury story), child PCS, child behaviour and socioemotional skills, child social cognition and competence, child sleep and fatigue, child quality of life, family functioning, parent mental health and parent quality of life (table 1). When both parents/legal guardians are present and willing to complete questionnaires independently, they will be encouraged to do so, and their scores will be averaged provided the correlations between them are sufficiently high. Primary caregivers will also provide a hair sample for stress measurement (when possible).

\section{Measures}

Measures completed by the child

Child assessment protocols will encompass three main age bands: 6-11.99 months, 12-23.99 months and 24-71.99 months. Details of the child measures included for each age band and the timepoints at which they will be administered are presented in table 1.

\section{Injury Story $\left(T_{2}\right)$}

Children aged 24 months and older will be asked a series of questions about their injury (mTBI and OI groups). The verbatim recording of their answers will be analysed using qualitative content and theme analysis in order to identify common mechanisms and reactions to concussion in young children and memory for the event.

\section{Global Development $\left(T_{3}\right)$}

Brigance Early Childhood Screens III $^{6162}$ : This screen will be used to assess child development in all age groups using 81-85 items for descriptive purposes, providing a standardised Total Score and subscores for motor development, language, cognitive and adaptive skills.

\section{Language (executive aspects, $T_{3}$ )}

Speeded Naming (Developmental NEuroPSYchological Assessment, NEPSY-II) ${ }^{63}$ : This test assesses rapid semantic access to and production of names of colours, shapes, sizes, letters or numbers in children older than 36 months of age. Children are shown an array of stimuli and are asked to name them in order as quickly as possible. Children receive 1 point for each correct answer. Total correct responses and total completion time are calculated.

Word Generation (NEPSY-II $)^{63}$ : This measure assesses verbal productivity through the ability to generate words within specific semantic and initial letter categories in children older than 36 months of age. Children are given a semantic or initial letter category and asked to produce as many words as possible in $60 \mathrm{~s}$. Children receive one point for each correct answer. Total orrect response is calculated.

\section{Executive Functions $\left(T_{2}\right.$ and $\left.T_{4}\right)$}

Minnesota Executive Function Scale ${ }^{64}$ : This adaptive assessment of global executive function is designed for children 24 months and older and will be administered individually on a touch-screen tablet. The total score (0-100 range) takes both trial accuracy and response time into account.

Bear and Dragon $\operatorname{task}^{65}{ }^{66}$ : This simplified version of the classic game "Simon Says" includes 10 items assessing inhibition in children 24 months and older. Children are instructed to do what the nice bear asks them (eg, 'touch your nose'), but not to do what the mean dragon 


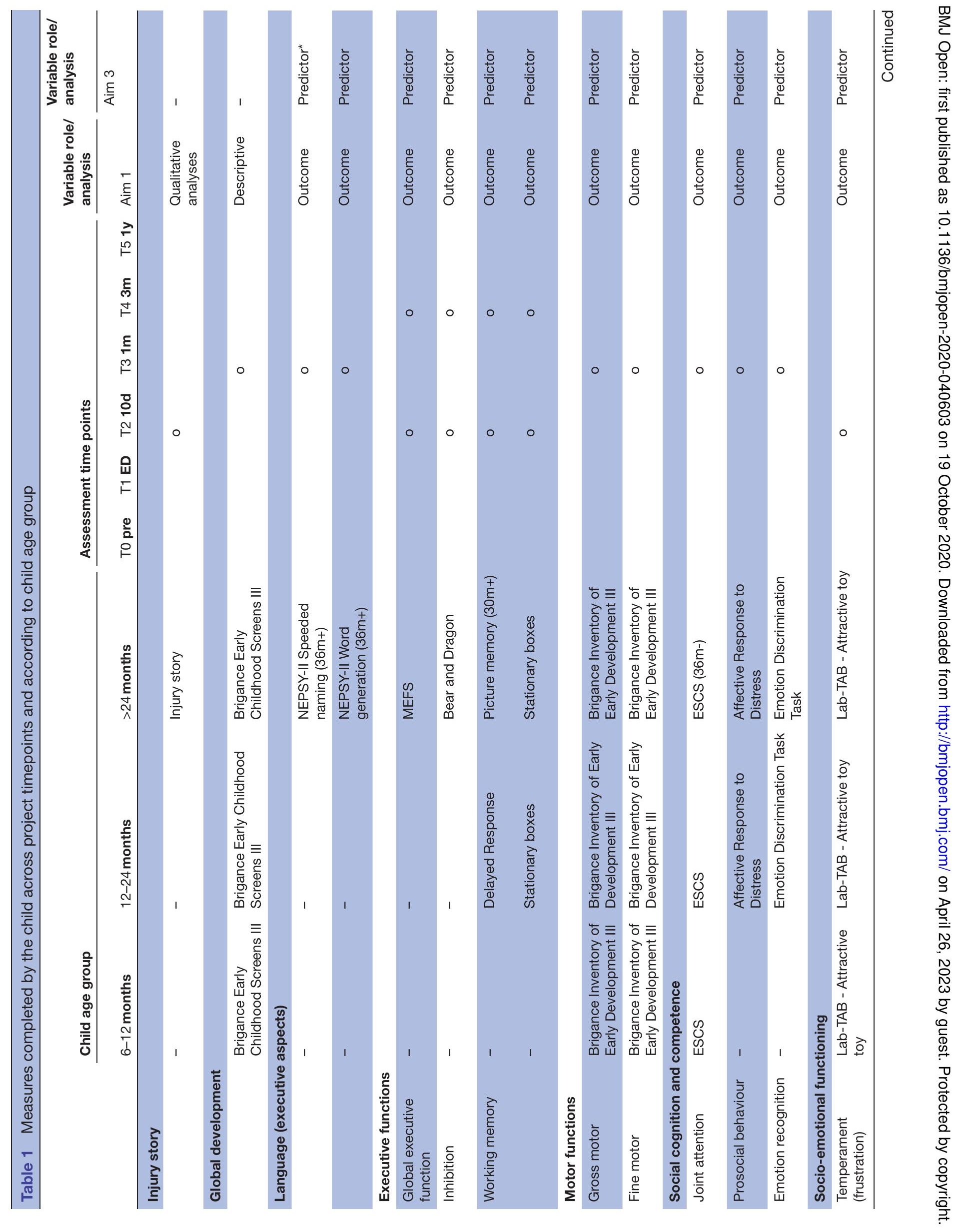




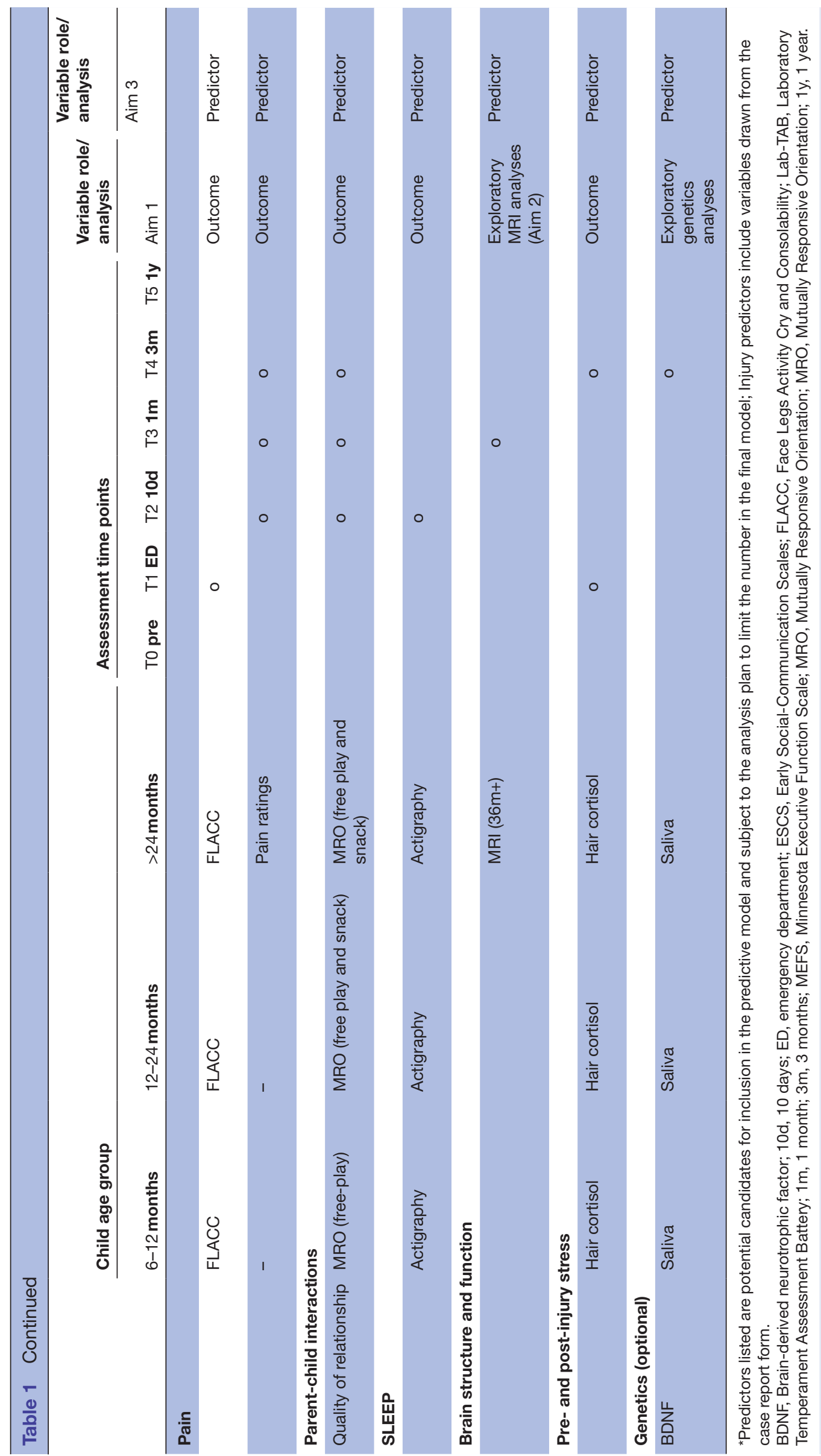

\begin{tabular}{l} 
W \\
\multirow{2}{c}{} \\
Oᄋ \\
$\frac{0}{8}$
\end{tabular}

$\stackrel{\vec{F}}{\stackrel{9}{+}}$

$\frac{\overline{0}}{\frac{\overline{0}}{\bar{\sigma}}}$

क

$\vec{\circ}$

$\overrightarrow{\vec{\omega}}$

옥.

$\stackrel{1}{\infty}$

N

六

के 
commands. Scores range from 0 (a full commanded movement) to 3 (no movement) on each dragon trial. Higher total score indicates better inhibitory control.

Delayed Response Task ${ }^{67}$ : This task includes 10 items to assess working memory in children between the ages of 12 and 24 months. A toy is hidden under one of two cups, and children are instructed to find the toy after a 5 s delay. Children receive 1 point for each correct reach. The correct response total score corresponds to the sum of the correct reaches.

Picture Memory task (Wechsler Preschool and Primary Scale of Intelligence, WPPSI-IV $)^{69}$ : In this 35 item workingmemory test, children 30 months and older view a stimulus page of one or more pictures for a specified time and then select the pictures from options on a response page. Pictures that children have seen earlier in the test may be repeated, so children have to remember if this was something they just saw, or something they saw earlier on a different question. Children receive 1 point for each correct answer. Higher total score reflects higher working memory performance.

Stationary Boxes Task ${ }^{68}$ : This task assesses working memory in children aged 12 months and older. Children are required to keep track of the location where they have previously searched for a reward from a fixed array of identical boxes (3, 6 and 9 boxes for children 12-30, 31-59 and 60+ months). Search efficiency score (number of boxes/total number of reaches) and perseverative errors ratio (number of reaches to a previous location on consecutive trials/number of boxes) are calculated.

\section{Motor Functions $\left(T_{3}\right)$}

Brigance Inventory of Early Development III ${ }^{61}$ : The Motor subsets from the inventory will be used to assess gross and fine motor skills in all children.

\section{Social cognition and competence $\left(T_{3}\right)$}

Early Social-Communication Scales (ESCS) ${ }^{70}$ : A subset of the observational ESCS protocol will be used to explore initiation of and response to joint attention in children younger than 36 months. The examiner presents a series of toys and tasks and attempts to engage the children to elicit socialcommunication skills. Behavioural ratings are made from videotaped sequencing according to the ESCS coding guidelines.

Infants' Affective Response to Distress ${ }^{71}{ }^{72}$ : In this observational measure designed for children 24 months and older, the examiner pretends to hurt him/herself during an interaction with the children, and the children's affective response to the examiner's distress is scored on a four-point Likert scale ( $0=$ "no expression of distress or concern' to 3 = 'strong indications of concern, worry or distress').

Emotion-Discrimination $\operatorname{task}^{73}$ : In this test of facial emotion recognition in children 24 months and older, a sheet of paper portraying two facial expressions (eg, happy vs sad) is presented to the children. Children are then handed six cards one by one (eg, three happy and three sad) and asked to place each card on the similar expression on the sheet of paper. The number of cards correctly placed measures the child's ability to discriminate facial expressions of emotion.

\section{Socioemotional Functioning (temperament, $T_{2}$ )}

Laboratory Temperament Assessment Battery-Attractive Toy subtest (Lab-TAB) ${ }^{74}$ : In this subtest, the child's response to frustration is measured observationally by placing a desired toy behind a plexiglass barrier $(<36$ months) or in transparent Box ( $\geq 36$ months). Frustration is coded based on the intensity of struggle (verbal and physical action against the barrier or the box), Facial Anger, Facial Sadness and Distress or Protest Vocalisations.

\section{Pain $\left(T_{1}, T_{2}, T_{3}\right.$ and $\left.T_{4}\right)$}

FLACC ${ }^{59^{3}}$ scale $\left(\mathrm{T}_{1}\right)$ : This scale is a behavioural observation measure of pain in infants and children of all ages. The FLACC includes behavioural categories and a variety of descriptors that are reliably associated with pain in children. This tool requires scoring of each of five behaviours on simple 0-2 ordinal scales, to provide a composite ordinal pain score of $0-10$.

Pain Ratings $\left(\mathrm{T}_{2}, \mathrm{~T}_{3}\right.$ and $\left.\mathrm{T}_{4}\right)$ : Pain intensity is assessed by asking children older than 24 months to point on a body diagram or on their own body where they have had aches or pains over the previous week. If they point to several areas, they are asked to identify which hurt the most. A 10-point faces rating scale (from very happy face corresponding to no pain, to crying face corresponding to very painful) is used to rate how much pain they felt.

\section{Parent-child interactions $\left(T_{2}, T_{3}\right.$ and $\left.T_{4}\right)$}

Mutually Responsive Orientation scale (MRO) ${ }^{75} 76$ : The quality of parent-child relationships will be assessed observationally via $10 \mathrm{~min}$ videotaped interaction sequences (<12 months: free pay; $\geq 12$ months: free play and snack). The videotaped interactions will be coded with regard to the dyadic nature of the parent-child exchanges across three subscales (Harmonious Communication, Mutual Cooperation and Emotional Ambiance). An MRO Total score is obtained by averaging the three MRO subscales.

\section{Sleep $\left(T_{2}\right)$}

Actigraphy: This method allows an objective assessment of sleep-wake cycles in young children. ${ }^{77}$ Children will be provided with an actigraph (Philips Respironics Actiwatch-2) to wear on their wrist (first choice) or ankle (second choice), 24 hours a day except when bathing for a total of 7 days. Actigraphs provide valid and reliable measures of the sleep-wake cycle in infants and young children. ${ }^{45}$ Children will be encouraged to wear the actigraph for seven consecutive days; however, data will be used regardless of the number of days the child ultimately wears the actigraph. ${ }^{78}$

Brain structure and function $\left(T_{3}\right)$

MRI: Advanced reseach MRI examnations will be performed in a subgroup of study participants, namely 
Table 2 KOALA MRI protocol acquisition parameters

\begin{tabular}{|c|c|c|c|c|c|c|c|c|c|}
\hline & Sequence & Echo time & $\begin{array}{l}\text { Repetition } \\
\text { time }\end{array}$ & $\begin{array}{l}\text { Inversion } \\
\text { time }\end{array}$ & $\begin{array}{l}\text { Flip } \\
\text { angle }\end{array}$ & FOV & $\begin{array}{l}\text { Slice } \\
\text { thickness }\end{array}$ & $\begin{array}{l}\text { Slice } \\
\text { spacing }\end{array}$ & Plane \\
\hline 1 & T1-weighted anatomic MRI & & & 540 & 12 & 23 & 0.9 & - & Axial \\
\hline 2 & $\begin{array}{l}\text { Quantitative susceptibility } \\
\text { mapping }\end{array}$ & Min full & Minimum & - & 10 & 24 & 1.9 & - & Axial \\
\hline 3 & $\begin{array}{l}\text { Diffusion-weighted imaging } 30 \\
\text { directions }\end{array}$ & Minimum & 6750.0 & - & - & 20 & 2.2 & 0.0 & Axial \\
\hline 4 & $\begin{array}{l}\text { Susceptibility-weighted } \\
\text { imaging }\end{array}$ & Min full & 2600.0 & - & 40 & 20 & 2.5 & 0.0 & Axial \\
\hline 5 & T2-weighted anatomic MRI & Min full & 450.0 & - & 30 & 23 & 3.6 & 0.0 & Oblique \\
\hline 6 & Resting-state functional MRI & 30.0 & 2000.0 & - & 60 & 23 & 3.6 & 0.0 & Oblique \\
\hline
\end{tabular}

Children will watch a movie of their choice throughout the MRI acquisition protocol.

KOALA, Kids' Outcomes And Long-term Abilities; MRI, Magnetic Resonance Imaging.

those between 36 and 72 months of age. All participants of this age without any contraindications to MRI will be invited to complete MRI either on the same day as their $\mathrm{T}_{3}$ follow-up (1-month postinjury) or on a separate day within a window of \pm 14 days depending on scanner and family availability. MRI sequences will be acquired without sedation after participation in a behavioural familiarisation paradigm developed by Frayne et $a l^{79}$ and adapted and validated by our team. ${ }^{80}$ As part of this paradigm, children watch a movie during image acquisition. MR images will be acquired on a 3T GE scanner using a 32 Channel matrix head coil at either the ACH or CHUSJ sites. The sequences acquired include T1-weighted anatomic MRI, quantitative susceptibility mapping (QSM, from which SWI will be derived), diffusion-weighted imaging, T2-weighted anatomic MRI and resting-state functional MRI (see table 2 for complete sequence parameters). This last sequence will occur while the participants continue to watch the movie.

\section{Preinjury and postInjury Child Stress ( $T_{1}$ and $\left.T_{4}\right)$}

Hair sample: Stress hormones levels (ie, cortisol) that accumulate in hair samples provide a measure of stress response over time in humans. ${ }^{81}$ A $3 \mathrm{~cm}$ hair sample of approximately $7.5 \mathrm{mg}$ will be obtained from children, when possible, during their ED visit $\left(\mathrm{T}_{1}\right)$. This sample will represent approximately 3 months of pre-injury accumulation of cortisol. A second sample will be obtained 3 months postinjury $\left(\mathrm{T}_{4}\right)$, representing approximately 3 months of postinjury accumulation of cortisol. Hair samples will be stored in aluminium foil until they are batch sent for analysis.

\section{Genetics $\left(T_{3}\right)$}

Saliva: This is an optional portion of the study. Children will be asked to provide a saliva sample $(2.5 \mathrm{~mL})$ in person at $\mathrm{T}_{3}$ using an assisted saliva collection tube (Oragene DNA, model OG-575). Samples will be identified based only on a study ID number. Initial analyses will focus on the brain-derived neurotrophic factor (BDNF) val66met polymorphism to replicate the most common investigations performed in older children with $\mathrm{TBI}^{82}$; however, remaining samples will be biobanked for further analyses on relevant genetic markers.

\section{Measures completed by parents}

Parent questionnaires are selected to ensure validity in the three study age bands . Details of the parent questionnaires included for each age band and the timepoints at which they are administered are presented in table 3 .

\section{Demographic information and medical history $\left(T_{2}\right.$ and $\left.T_{3}\right)$}

ABCs Laboratory Sociodemographic Questionnaire $\left(T_{3}\right)$ : This questionnaire provides information on the child's sex, handedness, ethnicity, birth characteristics, and developmental and medical history, as well as parent education, income and family characteristics. These variables will be used for descriptive and predictive purposes.

Injury story $\left(\mathrm{T}_{2}\right)$ : Parents of children (all ages) with mTBI or OI will be asked several questions related to the mechanism of their child's injury and their verbatim answers will be analysed using qualitative content and theme analysis in order to identify common mechanisms and reactions to concussion in young children and memory for the event.

\section{Child PCS}

REACTIONS(all timepoints) ${ }^{58}$ : This 106 item observational questionnaire is designed to document manifestations of common concussive symptoms in early childhood. Parents are asked to report whether their child verbalises symptoms typically associated with concussion and to indicate whether the child manifests behaviours that could be associated with these symptoms. Three domains of PCS are considered: cognitive (attention/concentration, memory, thinking or moving slowly), physical (headache, nausea, balance, fatigue, sleep, vision, sensitivity to light/noise/touch) and behavioural (irritability, mood and motivation, anxiety, regression, comfort).

Glasgow Outcome Scale-Extended Peds $\left(\mathrm{T}_{4} \text { and } \mathrm{T}_{5}\right)^{83}$ : For all age groups, global outcome and severity of TBI will be assessed in a semistructured interview resulting in 


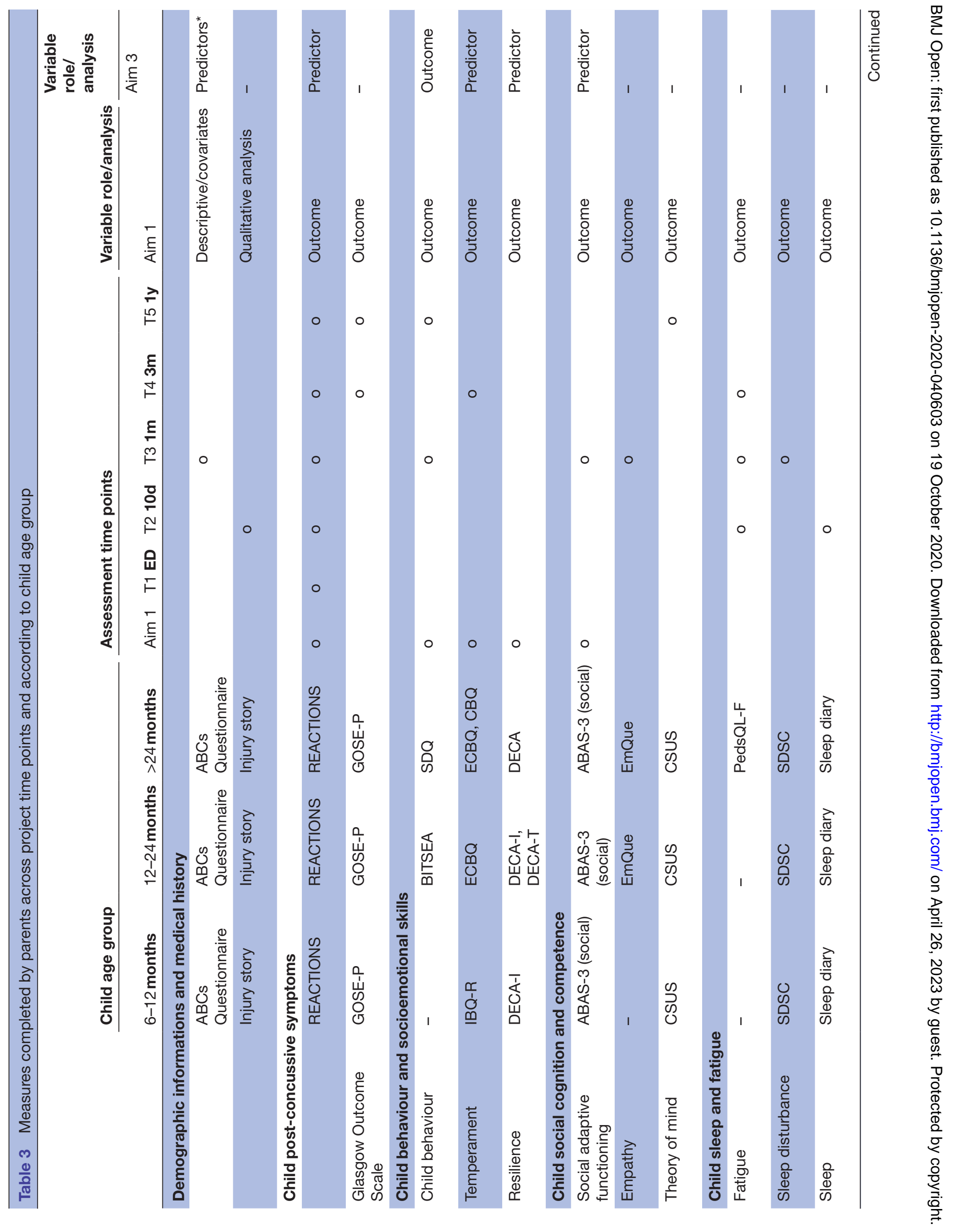




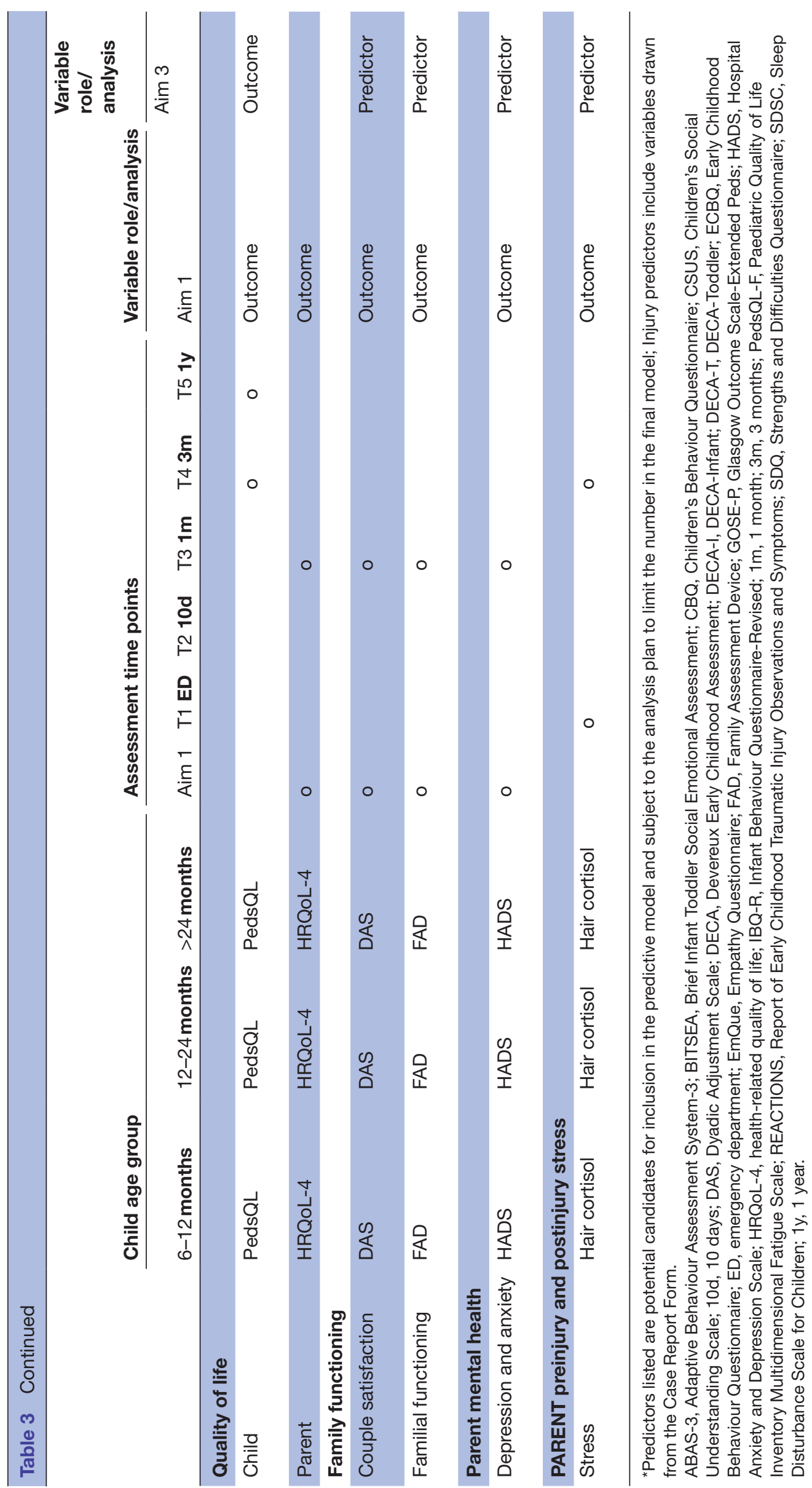

\begin{tabular}{l}
$\frac{0}{3}$ \\
$\frac{1}{2}$ \\
0 \\
0 \\
\hline
\end{tabular} 
an eight category classification: Dead, Vegetative State, Lower Severe Disability, Upper Severe Disability, Lower Moderate Disability, Upper Moderate Disability, Lower Good Recovery and Upper Good Recovery.

\section{Child Behaviour and Socioemotional Skills $\left(T_{0}, T_{3}, T_{4}\right.$ and $\left.T_{5}\right)$}

Brief Infant Toddler Social Emotional Assessment ${ }^{8485}\left(\mathrm{~T}_{0}\right.$, $\mathrm{T}_{3}$ and $\mathrm{T}_{5}$ ): This 42-item questionnaire will be used in children 12-24 months of age to measure social emotional problems (externalising problems, internalising problems, problems of dysregulation maladaptive behaviours, atypical behaviours) and competencies (attention, compliance, mastery motivation, prosocial peer relations, empathy, imitation/play skills, social relatedness) rated on a 3 -point scale $(0=$ not true/rarely, $1=$ sometimes true/sometimes and 2=very true/often). Responses are summed for each scale.

Strengths and Difficulties Questionnaire ${ }^{86}{ }^{87}\left(\mathrm{~T}_{0}, \mathrm{~T}_{3}\right.$ and $\mathrm{T}_{5}$ ): This 25-item questionnaire measures emotional symptoms, conduct problems, hyperactivity/inattention, peer relationship problems and prosocial behaviour in children 24 months and older on a 3-point scale $(0=$ "not true", 1 = "sometimes true" and 2 = "certainly true"). The score for each scale is the sum of item scores, generating a scale score from 0 to 10 . A total difficulties score (from scores for hyperactivity, emotional symptoms, conduct problems and peer problems) ranges from 0 to 40 .

Infant Behaviour Questionnaire-Revised/Early Childhood Behaviour Questionnaire/Children's Behaviour Questionnaire $^{889}\left(\mathrm{~T}_{0}\right.$ and $\left.\mathrm{T}_{4}\right)$ : Each of these age appropriate 36 -item questionnaires rates child temperament (all age groups) on a 7-point Likert scale (from 'extremely untrue of your child' to 'extremely true of your child'). Three dimensions of temperament are derived: Surgency, Negative Affectivity and Effortful Control.

Devereux Early Childhood Assessment (DECA-Infant, DECA-Toddler, DECA-Preschoolers $)^{90}{ }^{91}\left(\mathrm{~T}_{0}\right)$ : These age appropriate 36-38 item questionnaires will be used in all age groups to assess positive behaviours (strengths) typically seen in resilient children. Three protective factor scales are derived: Initiative (child's ability to use independent thought and actions to meet her or his needs), Attachment/Relationships (mutual, strong, long lasting relationship between the child and significant adults) and Self-Regulation (not in infant version, child's ability to gain control of and manage emotions, and sustain focus and attention). A Total Protective Factors scale provides an overall indication of the strength of the child's protective factors.

\section{Child Social Sognition and Competence $\left(T_{0}, T_{3}\right.$ and $\left.T_{5}\right)$}

Adaptive Behaviour Assessment System- $3^{92}\left(\mathrm{~T}_{0}\right.$ and $\left.\mathrm{T}_{3}\right)$ : The 24-item Social subscale provides a comprehensive assessment of everyday social adaptive functioning in children as young as 6 months of age. The caregiver indicates the frequency at which a behaviour is demonstrated on a 4-point scale (from $0=$ Is not able, to $3=$ Always when needed).
Empathy Questionnaire $^{93}\left(\mathrm{~T}_{3}\right): 20$ items representing three facets of empathy observable in children older than 12 months (Emotion Contagion, Attention to others' emotions, Prosocial responses to others' emotions) are rated in terms of the degree to which each item applies to the child on a 3 -point scale $(0=$ never, $1=$ sometimes, $2=$ often $)$. A total score of empathy includes all 20 items.

Children's Social Understanding Scale ${ }^{94}\left(\mathrm{~T}_{5}\right)$ : 18 items assess theory of mind abilities in children as young as 6 months. Items are answered on a scale from ' 1 Definitely Untrue' to '4 Definitely True'). A total score corresponds to the mean of all items.

\section{Child Sleep and Fatigue $\left(T_{2}, T_{3}\right.$ and $\left.T_{4}\right)$}

Paediatric Quality of Life Inventory Multidimensional Fatigue Scale (PedsQL $)^{95}\left(\mathrm{~T}_{2}, \mathrm{~T}_{3}\right.$ and $\left.\mathrm{T}_{4}\right): 18$ items measure signs of fatigue in children 24 months and older across three subscales (General Fatigue, Sleep/Rest Fatigue, Cognitive Fatigue) rated on a 5-point scale from 0 (never) to 4 (almost always).

Sleep Disturbance Scale for Children ${ }^{96}\left(\mathrm{~T}_{3}\right): 26$ items categorise sleep disorders in children as young as 6 months on a 5-point scale (from 'never' to 'always') across six disorder subdomains (initiating and maintaining sleep, sleep breathing, arousal, sleep-wake transition, excessive somnolence, sleep hyperhidrosis). The Total sleep disturbance score corresponds to the sum of the six factor scores. Higher scores reflect greater sleep disturbance.

Child Behaviour Checklist Sleep Problems Scale ${ }^{97}\left(\mathrm{~T}_{3}\right)$ : This parent-report behaviour questionnaire includes a Sleep Problems scale generated by summing up scores on the seven sleep items: (1) 'Does not want to sleep alone,' (2) 'Has trouble getting to sleep,' (3) 'Nightmares,' (4) 'Resists going to bed at night,' (5) 'Sleeps less than most children during day and/or night,' (6) 'Talks or cries out in sleep,' and (7) 'Wakes up often at night.' Parents are asked to describe their child's sleep-related behaviour on a 3-point Likert scale $(0=$ not true; $1=$ somewhat or sometimes true; $2=$ very true or often true).

Sleep diary $\left(\mathrm{T}_{2}\right)$ : During a total of 7 days, parents will be asked to indicate for each half-hour of the day whether the child was awake or asleep, and where he or she slept (eg, child's bedroom, car) or did not wear the actigraph (eg, bath), and to report any unusual events (eg, illness). This assessment is concurrent with child's sleep assessment using actigraphy.

Quality of life $\left(T_{0}, T_{3}, T_{4}\right.$ and $\left.T_{5}\right)$

Health-Related Quality of Life (HRQoL- 4$)^{98}\left(\mathrm{~T}_{0}\right.$ and $\mathrm{T}_{3}$ ): 4 items measure parental quality of life via a self-report of global health including a measure of days considered to be 'unhealthy' and an estimate of the number of days on which poor physical or mental health kept the respondent from engaging in his or her typical daily activities.

PedsQL $4.0^{99}\left(\mathrm{~T}_{4}\right.$, and $\left.\mathrm{T}_{5}\right)$ : 21-25 items measure HRQoL in children as young as 6 months on a 5-point scale (from $0=$ never to $4=$ almost always) across four domains (physical, emotional, social, school). It provides a total scale 
score $(0-100)$, a physical health summary score and a psychosocial health summary score.

\section{Family Functioning ( $T_{0}$ and $\left.T_{3}\right)$}

Dyadic Adjustment Scale ${ }^{100101}$ : 4 items measure couple satisfaction. Three items are on a 6-point Likert scale ranging from 0 (all the time) to 5 (never), while the final item is on a 7-point scale ranging from 0 (extremely unhappy) to 6 (perfect). The Total Score corresponds to the mean of all items. Higher scores reflect higher couple satisfaction.

Family Assessment Device ${ }^{102}: 12$ items measure family adjustment rated on a 4-point scale corresponding to the degree to which the statement describes the family (1=strongly agree, 2=agree, 3=disagree, 4=strongly disagree). The total score corresponds to the mean of all items. Higher scores indicate poorer family functioning.

\section{Parent Mental Health $\left(T_{0}\right.$ and $\left.T_{3}\right)$}

Hospital Anxiety and Depression Scale ${ }^{103}: 14$ items assess anxiety and depression in adults on a scale from 0 (Not at all) to 3 (Most of the time) resulting in a depression score, an anxiety score, and a total score. Higher scores indicate more symptoms.

\section{Preinjury and Postinjury Parent Stress ( $T_{1}$ and $\left.T_{4}\right)$}

Hair sample: A $3 \mathrm{~cm}$ hair sample of approximately 7.5 $\mathrm{mg}$ will be obtained from parents, when possible, during their ED visit $\left(T_{1}\right)$ representing approximately 3 months of preinjury accumulation of cortisol; and 3 months postinjury $\left(\mathrm{T}_{4}\right)$, representing approximately 3 months of postinjury accumulation of cortisol. Parent stress levels will be studied in associated with child stress levels as well as behavioural and cognitive recovery given previous evidence that parent stress may impact outcome after TBI. ${ }^{104}$

\section{Data management and privacy protection}

\section{Database}

The database will be maintained in Research Electronic Data Capture (REDCap), a web-based platform that allows remote data entry from each site. All data will be entered directly into REDCap whenever possible. Data, that is, transcribed (eg, all observational child measures that require remote scoring and/or qualitative analysis) will be scored offline and then entered into REDCap. All data will undergo quality checks using procedures such as double verification and range and error checks. Data from all sites will be merged in a shared central REDCap database stored at the CHUSJ site. All video data (observational measures) and MRI data will be transferred to the CHUSJ main study site using a secure pipeline for analysis.

All measures will be collected only by study investigators or research personnel to ensure the security and deidentification of the data. Only members of the research team will be able to access the REDCap database, and will use individual logins and passwords with double verification. All biological material (hair, saliva) will be collected and kept according to standard operating and ethical procedures.

\section{Analysis plan}

Group differences on main predictor/outcome variables (aim 1)

The first aim of the study is to document the consequences of preschool mTBI on PCS, motor, cognitive, social and behavioural functioning, as well as psychobiological markers (sleep, stress).

Multivariable longitudinal modelling strategies (eg, linear mixed effects models or generalised least squares) will be applied to estimate group differences (mTBI vs OI vs CC) at relevant follow-up time-points for each outcome. Each outcome will be operationalised as continuous/ordinal variables and specified as the dependent variable in separate statistical models. To minimise the risk of confounding bias, a variable selection strategy will be used to ensure that the most important covariates are ultimately included for adjustment in each fitted model. A series of steps will be conducted towards a final model for each outcome. First, univariate regressions will be initially applied to screen candidate predictors where $\mathrm{p}<0.2$ will be kept (see tables 1 and 3 , for list of candidate predictors). Second, correlation among candidate predictors will be performed and if $r>0.7$ is found, the variable with the stronger association with outcome will be kept. Third, multiple imputation strategies will be applied to the dataset at this stage such that participants providing incomplete covariate information can still contribute to the next and final step in this process. Multiple imputation will be applied to estimate missing data ${ }^{105}$ using the Markov Chain Monte Carlo procedure. Twenty imputations will be used to maximise the precision of imputed data. ${ }^{106} 107$ The imputations will be performed by comprehensive inclusion of auxillary variables when building imputation models. In the last step, a forward stepwise selection proceduce will be applied to arrive at a final model (for each outcome) containing the interaction between the 3-level group factor and time as a fixed predictor, and covariates demonstrating the strongest associations with the outcome (ie, $\mathrm{p}<0.05$ ) up to the allowable limit based on considerations to the 10 'eventsper-variable' rule of thumb.

\section{Genetics}

Amplification will be performed using a thermal cycler (Biometra Tprofessional) using a PCR approach, with the following oligonucleotide primer pairs: 5'-biotin before GGACTCTGGAGAGCGTGAAT-3 and 5'-reverse CGAACTTTCTGGTCCTCATC-3'. In addition to buffers, nucleotide components and a dose of $0.01 \mathrm{U}$ of Taq polymerase supplier of PCR Master Mix (Qiagen), the amplification reactions contain $1 \mu \mathrm{g}$ of DNA derived from saliva, $1 \mu \mathrm{M}$ each primer, $0.4 \mathrm{mM}$ of dNTP, $1.0 \mathrm{mM}$ $\mathrm{MgCl} 2$, in a final volume of $50 \mu \mathrm{L}$. The PCR conditions 
include 35 cycles: $30 \mathrm{~s}$ at $95^{\circ} \mathrm{C} ; 30 \mathrm{~s}$ at $61.2^{\circ} \mathrm{C}$; and $1 \mathrm{~min}$ at $72^{\circ} \mathrm{C}$. These 35 cycles of amplification will be preceded by an initial heating step of $3 \mathrm{~min}$ at $95^{\circ} \mathrm{C}$ and followed by a final extension of $4 \mathrm{~min}$ at $72^{\circ} \mathrm{C}$. The PCR products are visualised on a $1.2 \%$ agarose gel. The Val66Met polymorphism will be sequenced with a routine pyrosequencing protocol ${ }^{108}$ with a slight modification using the sequencing oligomer 5'- GCTGACACTTTCGAACA -3'. The sequence to be analysed is: CA / GTGATAGAAGAG. Other candidates genes involved in neurotrauma response neural repair and plasticity may be subsequently studied depending on availability of saliva sample.

\section{Stress}

Stress will be evaluated by Hair Cortisol Concentration determined via liquid chromatography tandem mass spectrometry (Dresden Labservice, Germany). roup and repeated measures analyses will be used to determine change in cortisol levels between the two timepoints (Hair sample taken in ED as a preinjury measure and hair sample 3 months postinjury) and between groups.

\section{Sleep}

Actigraphy data will be collected using AW-2 actigraphs in 1 min epochs and analysed using the Phillips Respironics Actiware software V.5.70. The automated manufacturer's low sensitivity threshold algorithm (80 activity counts per epoch), which has been shown to be appropriate for preschoolers, ${ }^{77109}$ will be used to determine the minuteby-minute sleep-wake status. To corroborate actigraphy data and identify potential artefacts, the sleep diary information for the 7 days will be used during which the child wore the actigraph. When the child had an atypical night, was not wearing the actigraph, or was cosleeping, nights will be excluded on a case-by-case basis. Sleep onset and sleep offset will be determined based on visual examination of the actogram and guided by the diary. Actigraphic variables will be derived across the available assessment period: night-time sleep duration (total number of minutes from sleep onset to sleep offset that were scored as sleep) and sleep efficiency (sleep minutes at night / (sleep minutes at night + wake min at night) $* 100)$. Group comparisons will be performed on actigraphic variables to determine whether mTBI affects sleep indicators in comparison to OI or CC groups.

Neuroimaging analyses (aim 2)

MRI quality control

All MRI images will be visually inspected by a trained researcher for artefacts and image quality to ensure they are of sufficient quality to be analysed. Motion correction will be applied with adapted processing depending on the MRI sequence. For resting-state analyses, motion parameters will be added as a nuisance covariates at first-level analyses.

\section{MRI coding for visible lesions}

T1 and QSM (converted to SWI) sequences will be read and coded by a radiologist blind to group (mTBI vs OI vs
CC) using a standard form for documenting lesion location and type.

\section{Preprocessing and analyses}

Neuroimaging preprocessing and analyses will be performed using standard neuroimaging software (eg, SPM12, FreeSurfer, FSL). Processing adapted for paediatric neuroimaging data, particularly use of paediatric templates, will be preferred. Initial neuroimaging analyses will focus on group comparisons (mTBI vs controls) to examine differences between children with and without history of mTBI in brain morphometry (grey and white matter volumes, cortical thickness and gyrification, using 3D T1 sequence), white matter microstructure (using DTI data, including fractional anisotropy and mean diffusivity as outcome measures), and functional connectivity in the default mode network (using resting state data). Other analytic approaches may be incorporated as appropriate and as new tools emerge.

\section{Predictive models (aim 3)}

To predict global functional outcomes after mTBI, separate multivariable models will be fit with either the 1-month or 3-month outcome as the dependent variable. The rationale for this is that we hypothesise unique predictors for outcomes measured at the different time points, and in the case of 3-month outcomes, we also plan to specifically incorporate several 1 month measures as candidate predictors in addition to variables measured at prior time points. All outcomes will be operationalised as continuous variables and all final models will be developed based on the following general procedure. Initially, univariate linear regression will be used to screen all candidate variables for entry $(p<0.20)$ into multivariable models. Multiple imputations will be performed at this stage, following the approach described above aim 1 . Then, multiple hierarchical regression will establish the contribution of host (eg, child age, sex, genetics (BDNF), premorbid temperament, resilience, adaptive skills, behaviour, cognition), socioenvironmental (eg, parent mental health, family functioning, parent-child interactions, SES), injury (eg, injury severity markers, PCS, neuropathology) and psychobiological (eg, stress, sleep) factors to global outcomes of interest (eg, PCS, behaviour, quality of life), by entering univariate predictors into four blocks (child (host), socioenvironmental, injury, psychobiological variables). As a final step, backwards elimination will be employed such that only predictors with the strongest statistical associations with the outcome remains, to keep within the 10:1 event-per-variable ratio in the final model. Interactions among retained predictors will be considered if theoretically plausible and there is sufficient df after prioritising the inclusion of main effects in the above procedure.

\section{Qualitative analyses}

Injury story verbatims will be transcribed verbatim and then coded for common themes. Coding will be done 
on the interview transcripts using QSR Nvivo (Doncaster, Australia) qualitative analysis software. A coding guide will be developed using a classic qualitative and thematic content analysis approach. ${ }^{110}$ This coding guide, which will be used to structure the coding, will be developed on the basis of the analysis of a small sample of interviews (5), from which free nodes will be generated in an open coding phase. Throughout the coding process, modifications and additions will be made to the coding guide and nodes as each interview is analysed. This will be done in constant comparison with previous coding so that the data can be analysed accurately. ${ }^{110}$ These frequent revisions will ensure the rigour and depth of the analysis, resulting in a final coding guide containing the codes (nodes) and their definitions. The coding guide will consist of primary, secondary and tertiary nodes (or codes). Each interview will be coded in its entirety, and each element may be coded in more than one node at a time. The coding will involve discussions within the research team to reduce subjective bias on the part of the coder. A consensus check will be carried out for all coding as well as for the coding structure.

\section{Statistical power}

The objectives for participant recruitment were established primarily to have sufficient power for group difference analyses and regression models planned to meet objectives 1 and 3. For objective 1, the main consideration was to have sufficient power to detect a group differences in the context of a well-adjusted multivariable regression. Since meaningfully important score differences and expected variability for many of our proposed outcomes remain unclear, we are using the Cohen's conventional medium effect size as the basis for our sample size calculation. If we reduce the problem to a simplistic case of a balanced, one-way analysis of variance, at an alpha of 0.05 and power of 0.9 , the sample size required per group to detect a medium effect size $(\mathrm{f}=0.25)$ is 69 . However, as we are planning comprehensive covariate adjustment in our regression models (ie, include the maximum allowable covariates), this approximation will be conservative since covariate adjustment is expected to markedly improve the precision of the estimated group differences (ie, increases the power and reduces the needed sample size). Thus, the proposed sample size of 300 (150 mTBI, 75 OI, $75 \mathrm{CC}$ ) is expected to provide adequate power for the study, leaving leeway for sample attrition (estimated at between $85 \%$ and $65 \%$ over the 12 months follow-up). Our intention to apply multiple imputation strategies to the dataset also minimises the potential need to discard observations from the final fitted models due to incomplete data. For objective 3, the main consideration was to ensure there is adequate $\mathrm{df}$ for a comprehensive multivariable linear regression model to predict outcomes following mTBI. At the expected recruitment of $150 \mathrm{mTBI}$ patients, this will afford $15 \mathrm{df}$ (according to 10:1 events-per-variable rule of thumb).
This is expected to be adequate as previous prediction models in the field have revealed no more than this number of major predictors for functional outcomes following mTBI. ${ }^{111-113}$ In addition, at this sample size and assuming 15 numerator df (ie, 15 predictors), this would enable $84 \%$ statistical power to detect a medium effect size of $\mathrm{f}^{2}=0.15$ (which translates to an $\mathrm{R}^{2}$ of 0.13 ).

\section{Potential limitations}

The evaluation of functioning in young children has inherent limitations. First, some children may not be able or willing to complete the full study assessment protocols at each time point. For the MRI portion, some children may not complete the full imaging protocol or may refuse to participate in this portion of the study. Second, the genetics portion of the study is exploratory and the sample size for this substudy is limited for conducting genome-wide analyses. We have chosen to focus on the BDNF polymorphisms to begin with because of the existence of literature supporting its role in the context of mTBI. The examiners will all be trained in conducting developmental and neuropsychological assessments and knowledgeable about ways to optimise participation in preschool children. The sample has some limitations in terms of generalisability. The study will reflect the outcomes of children whose parents seek care at large urban tertiary care hospitals, and therefore may not reflect those who either do not seek care or do so in family clinics or rural centres. Finally, 'early mTBI' lacks a consensual definition and clear diagnostic criteria, leaving some subjectivity in recruitement criteria. To guard including children who only have trivial head bumps, we require the traumatic accident to be witnessed, and, in addition to the analyses proposed, will explore group differences by stratifying the mTBI group to distinguish 'possible', 'probable', and definite' mTBI, as recommended by Kutcher and Giza. ${ }^{114}$

\section{Ethics and dissemination}

The study has been approved by all participating institutions (Sainte-Justine University Hospital Research Ethics Board, McGill University Health Centre Research Ethics Board, Univeristy of Calgary Conjoint Health Research Ethics Board). No significant risks are associated with study participation. The main concern associated with the direct child assessments is possible fatigue. Breaks will be provided as needed to study participants. Participation brings no direct benefit other than small compensations in the form of toys for the children and a small monetary compensation for parents. Parents also receive a brief research report including summary information on their child's performance on a select number of standardised measures used in the study. On occasion, direct child assessment or the responses of a parent in a questionnaire can suggest a developmental, cognitive, or psychological problem that requires clinical follow-up. Data will not be systematically screened 
for such problems as the assessments are not considered clinically comprehensive; however, if a significant problem is noted by a research team member, it will be brought to the attention of the PI (a neuropsychologist) who will advise on follow-up (ex: recommendation to family in study report for referral for a clinical assessment; suggest that the family consult family doctor or paediatrician).

MRI is not known to be associated with any significant risks or side effects, ${ }^{115}$ and participants (as well as their parents if present in the scanning room) all undergo thorough screening for contraindications to MRI acquisition. The behavioural familiarisation protocol used to introduce MRI to participants has been previously validated, ${ }^{79} 80$ and MRI acquisition will be aborted if a participant shows any signs of significant anxiety or discomfort before or during scanning. MRI images will not undergo systematic clinical evaluation and no clinical reports will be provided. The genes that will be studied are not diagnostic of any specific neurodevelopmental conditions and the limited literature in this area, especially in this age range, is insufficient to determine whether any genes studied in the context of the project can facilitate recovery or not. Therefore, parents will be advised that they will not be provided with any results of genetic testing. Parents will have the option to consent to any remaining biological samples to be stored in a biobank for future analyses, otherwise, any remaining samples will be destroyed.

The programme will rely on scientific dissemination through open access journals and repositories and an integrated knowledge translation plan designed at the study outset in consultation with an expert knowledge broker. Partners are organisations focusing on clinical practice and neurodevelopment and their respective networks and knowledge users, include parents of infants, toddlers and preschool children, health professionals, health promotion organisations, day cares and community organisations focusing on early development, school boards, scientists and policy-makers.

\section{Significance}

Identifying the long-term consequences of early mTBI and their predictors has the potential to provide substantial benefits for children, their families and health professionals. In the short term, the project will allow us to understand how symptoms, motor, cognitive, social and behavioural skills evolve in the first year following early mTBI. This will identify specific loci for treatment and intervention in this distinct developmental group. The elaboration of predictive models will permit the identification of children who present specific risk factors for poor outcome. Associating the superior sensitivity of advanced neuroimaging techniques with a no-risk behavioural protocol for scanning young children could lead to improvements in the management, detection and diagnosis of young children with mTBI in the acute setting. In particular, the study will provide insight into typical PCS manifestations and patterns in young children. Study findings could promote the development of clinical guidelines for the management of early mTBI, as current guidelines typically do not apply to young children. The consolidation of basic skills in early childhood is known to be associated with later academic and social success, and early identification of problems and their rapid remediation is demonstrably effective in improving later functioning. ${ }^{116}$

\section{Author affiliations}

${ }^{1}$ Psychology, Université de Montréal, Montreal, Quebec, Canada

${ }^{2}$ Sainte-Justine Hospital Research Center, Montreal, Quebec, Canada

${ }^{3}$ Psychiatry, LMU München, Munchen, Bayern, Germany

${ }^{4}$ Psychology, University of Calgary, Calgary, Alberta, Canada

${ }^{5}$ Research Institute, Alberta Children's Hospital, Calgary, Alberta, Canada

${ }^{6}$ Hotchkiss Brain Institute, University of Calgary, Calgary, Alberta, Canada

${ }^{7}$ School of Physical and Occupational Therapy, McGill University, Montreal, Quebec, Canada

${ }^{8}$ Trauma, Montreal Children's Hospital, Montreal, Quebec, Canada

${ }^{9}$ Clinical Research Unit, Children's Hospital of Eastern Ontario Research Institute,

Ottawa, Ontario, Canada

${ }^{10}$ Pediatric Emergency Medicine, CHU Sainte-Justine, Montreal, Quebec, Canada

${ }^{11}$ Pediatrics, University of Calgary, Calgary, Alberta, Canada

${ }^{12}$ Pediatrics, Alberta Children's Hospital, Calgary, Alberta, Canada

${ }^{13}$ Pediatric Emergency Medicine, Montreal Children's Hospital, McGill University Health Center, Montreal, Quebec, Canada

${ }^{14}$ Psychology, Université de Montreal, Montreal, Quebec, Canada

${ }^{15}$ Radiology, University of Calgary, Calgary, Alberta, Canada

${ }^{16}$ Radiology, CHU Sainte-Justine, Montreal, Quebec, Canada

${ }^{17}$ Psychiatry, Université de Montréal, Montreal, Quebec, Canada

${ }^{18}$ Surgery, Université de Montréal, Montreal, Quebec, Canada

${ }^{19}$ Pediatrics, Children's Hospital of Eastern Ontario, Ottawa, Ontario, Canada

Acknowledgements We would like to thank the research coordinators and assistants at all of the three sites for their contributions to this project, in particular Maria Tran for overall coordination of the study. The proposed research is endorsed by the Pediatric Emergency Research Canada (PERC) network (https://www.perccanada.ca).

Contributors MHB, KY, IG, AB and FD designed the study with input from JG, AS, $\mathrm{BB}$ and $\mathrm{RZ}$ on the acute assessment procedures and participant criteria. MHB, $\mathrm{CL}, \mathrm{REJ}, \mathrm{MD}$ and SD guided the development of the neuroimaging protocol. MHB and $L d B$ developed the genetic component of the study. SL developed the cortisol collection and analysis strategy. MHB and KT developed the analysis plan. MHB wrote the first draft of the manuscript. All authors read, critically revised and approved the final version of the manuscript.

Funding This work was supported by the Canadian Institutes of Health Research grant number FDN148417.

Competing interests CL's spouse is an employee of General Electric Healthcare. The other authors report no biomedical financial interests or potential conflicts of interest.

Patient and public involvement See patient involvement statement in text.

Patient consent for publication Not required.

Provenance and peer review Not commissioned; externally peer reviewed.

Open access This is an open access article distributed in accordance with the Creative Commons Attribution Non Commercial (CC BY-NC 4.0) license, which permits others to distribute, remix, adapt, build upon this work non-commercially, and license their derivative works on different terms, provided the original work is properly cited, appropriate credit is given, any changes made indicated, and the use is non-commercial. See: http://creativecommons.org/licenses/by-nc/4.0/.

\section{ORCID iDs}

Miriam H Beauchamp http://orcid.org/0000-0002-8637-6361

Keith Yeates http://orcid.org/0000-0001-7680-2892 
Roger Zemek http://orcid.org/0000-0001-7807-2459

\section{REFERENCES}

1 McKinlay A, Grace RC, Horwood LJ, et al. Prevalence of traumatic brain injury among children, adolescents and young adults: prospective evidence from a birth cohort. Brain Inj 2008;22:175-81.

2 Rutland-Brown W, Langlois JA, Thomas KE, et al. Incidence of traumatic brain injury in the United States, 2003. J Head Trauma Rehabil 2006;21:544-8.

3 Crowe LM, Anderson V, Catroppa C, et al. Head injuries related to sports and recreation activities in school-age children and adolescents: data from a referral centre in Victoria, Australia. Emerg Med Australas 2010;22:56-61.

4 Marin JR, Weaver MD, Yealy DM, et al. Trends in visits for traumatic brain injury to emergency departments in the United States. JAMA 2014;311:1917-9.

5 Congeni J. Management of the adolescent concussion victim. Adolesc Med State Art Rev 2009;20:41-56.

6 Marcotte A-C. Orientations ministérielles pour le traumatisme cranio-cérébral. In: Traumtologie Pd, ed. Québec: Ministère de la Santé et Services Sociaux, 2010.

7 Kochanek PM. Pediatric traumatic brain injury: quo vadis? Dev Neurosci 2006;28:244-55.

8 Noppens R, Brambrink AM. Traumatic brain injury in children clinical implications. Exp Toxicol Pathol 2004;56:113-25.

9 Anderson V, Spencer-Smith M, Leventer R, et al. Childhood brain insult: can age at insult help us predict outcome? Brain 2009;132:45-56.

10 Yeates KO, Taylor HG, Walz NC, et al. The family environment as a moderator of psychosocial outcomes following traumatic brain injury in young children. Neuropsychology 2010;24:345-56.

11 Garcia D, Hungerford GM, Bagner DM. Topical review: negative behavioral and cognitive outcomes following traumatic brain injury in early childhood. J Pediatr Psychol 2015;40:391-7.

12 Ewing-Cobbs L, Prasad MR, Kramer L, et al. Late intellectual and academic outcomes following traumatic brain injury sustained during early childhood. J Neurosurg 2006;105:287-96.

13 Tlustos SJ, Chiu CY, Walz NC, et al. Emotion labeling and socioemotional outcomes 18 months after early childhood traumatic brain injury. J Int Neuropsychol Soc 2011;17:1132-42.

14 Crowe LM, Catroppa C, Babl FE, et al. Intellectual, behavioral, and social outcomes of accidental traumatic brain injury in early childhood. Pediatrics 2012;129:e262-8.

15 Haarbauer-Krupa J, King TZ, Wise J, et al. Early elementary school outcome in children with a history of traumatic brain injury before age 6 years. J Head Trauma Rehabil 2019;34:111-21.

16 Rogers A, McKinlay A. The long-term effects of childhood traumatic brain injury on adulthood relationship quality. Brain Inj 2019;33:649-56.

17 Anderson V, Catroppa C. Memory outcome at 5 years postchildhood traumatic brain injury. Brain Inj 2007;21:1399-409.

18 Ganesalingam K, Sanson A, Anderson V, et al. Self-Regulation as a mediator of the effects of childhood traumatic brain injury on social and behavioral functioning. J Int Neuropsychol Soc 2007:13:298-311.

19 Taylor HG, Swartwout MD, Yeates KO, et al. Traumatic brain injury in young children: postacute effects on cognitive and school readiness skills. J Int Neuropsychol Soc 2008;14:734-45.

20 Yeates KO, Swift E, Taylor HG, et al. Short- and long-term social outcomes following pediatric traumatic brain injury. J Int Neuropsychol Soc 2004;10:412-26.

21 Keenan HT, Hooper SR, Wetherington CE, et al. Neurodevelopmental consequences of early traumatic brain injury in 3-year-old children. Pediatrics 2007;119:e616-23.

22 Keenan HT, Presson AP, Clark AE, et al. Longitudinal developmental outcomes after traumatic brain injury in young children: are infants more vulnerable than toddlers? J Neurotrauma 2019;36:282-92.

23 Bellerose J, Bernier A, Beaudoin C, et al. When injury clouds understanding of others: theory of mind after mild TBI in preschool children. J Int Neuropsychol Soc 2015:1-11.

24 Bellerose J, Bernier A, Beaudoin C, et al. Long-Term brain-injuryspecific effects following preschool mild TBI: a study of theory of mind. Neuropsychology 2017;31:229-41.

25 D'Hondt F, Lassonde M, Thebault-Dagher F, et al. Electrophysiological correlates of emotional face processing after mild traumatic brain injury in preschool children. Cogn Affect Behav Neurosci 2017:17:124-42.

26 Gagner C, Landry-Roy C, Bernier A, et al. Behavioral consequences of mild traumatic brain injury in preschoolers. Psychol Med 2018;48:1551-9.
27 Lalonde G, Bernier A, Beaudoin C, et al. Investigating social functioning after early mild TBI: the quality of parent-child interactions. J Neuropsychol 2018;12:1-22.

28 Dégeilh F, Bernier A, Gravel J, et al. Developmental trajectories of adaptive functioning following early mild traumatic brain injury. Dev Psychobiol 2018;60:1037-47.

29 Beauchamp MH, Anderson V. Cognitive and psychopathological sequelae of pediatric traumatic brain injury. Handb Clin Neurol 2013;112:913-20.

30 Yeates KO, Taylor HG, Rusin J, et al. Premorbid child and family functioning as predictors of post-concussive symptoms in children with mild traumatic brain injuries. Int J Dev Neurosci 2012;30:231-7.

31 Anderson V, Catroppa C, Godfrey C, Rosenfeld JV, et al. Intellectual ability 10 years after traumatic brain injury in infancy and childhood: what predicts outcome? J Neurotrauma 2012;29:143-53.

32 Wade SL, Zhang N, Yeates KO, et al. Social environmental Moderators of long-term functional outcomes of early childhood brain injury. JAMA Pediatr 2016;170:343-9.

33 Narad ME, Treble-Barna A, Zang H, et al. Parenting behaviors after moderate - severe traumatic injury in early childhood. Dev Neurorehabil 2018:1-8.

34 Suskauer SJ, Huisman TA. Neuroimaging in pediatric traumatic brain injury: current and future predictors of functional outcome. Dev Disabil Res Rev 2009;15:117-23.

35 Beauchamp MH, Beare R, Ditchfield M, et al. Susceptibility weighted imaging and its relationship to outcome after pediatric traumatic brain injury. Cortex 2013;49:591-8.

36 Kassam I, Gagnon F, Cusimano MD. Association of the APOE- $\varepsilon 4$ allele with outcome of traumatic brain injury in children and youth: a meta-analysis and meta-regression. J Neurol Neurosurg Psychiatry 2016;87:433-40.

37 Botchway EN, Godfrey C, Anderson V, et al. A systematic review of sleep-wake disturbances in childhood traumatic brain injury: relationship with fatigue, depression, and quality of life. $J$ Head Trauma Rehabil 2019;34:241-56.

38 Gagner C, Landry-Roy C, Lainé F, et al. Sleep-Wake disturbances and fatigue after pediatric traumatic brain injury: a systematic review of the literature. J Neurotrauma 2015;32:1539-352.

39 Zaitsev M, Maclaren J, Herbst M. Motion artifacts in MRI: a complex problem with many partial solutions. J Magn Reson Imaging 2015;42:887-901.

40 Beauchamp MH, Ditchfield M, Babl FE, et al. Detecting traumatic brain lesions in children: CT versus MRI versus susceptibility weighted imaging (SWI). J Neurotrauma 2011;28:915-27.

41 Dennis EL, Babikian T, Giza CC, et al. Neuroimaging of the injured pediatric brain: methods and new lessons. Neuroscientist 2018;24:652-70.

42 Currie S, Saleem N, Straiton JA, et al. Imaging assessment of traumatic brain injury. Postgrad Med J 2016;92:41-50.

43 Gagner C, Tuerk C, De Beaumont L, et al. Brain-Derived neurotrophic factor Val66Met polymorphism and internalizing behaviors after early mild traumatic brain injury. $J$ Neurotrauma 2020.

44 Landry-Roy C, Bernier A, Gravel J, et al. Predictors of sleep outcomes following mild traumatic brain injury in preschoolers: subjective and objective assessment of outcome. J Head Trauma Rehabil 2017;32:E13-23.

45 Landry-Roy C, Bernier A, Gravel J, et al. Executive functions and their relation to sleep following mild traumatic brain injury in preschoolers. J Int Neuropsychol Soc 2018;24:769-80.

46 Ganesalingam K, Yeates KO, Ginn MS, et al. Family burden and parental distress following mild traumatic brain injury in children and its relationship to Post-concussive symptoms. J Pediatr Psychol 2008;33:621-9.

47 Stancin T, Wade SL, Walz NC, et al. Traumatic brain injuries in early childhood: initial impact on the family. J Dev Behav Pediatr 2008:29:253-61.

48 Durber CM, Yeates KO, Taylor HG, et al. The family environment predicts long-term academic achievement and classroom behavior following traumatic brain injury in early childhood. Neuropsychology 2017;31:499-507.

49 Mathias JL, Dennington V, Bowden SC, et al. Community versus orthopaedic controls in traumatic brain injury research: how comparable are they? Brain Inj 2013;27:887-95.

50 Beauchamp MH, Landry-Roy C, Gravel J, et al. Should young children with traumatic brain injury be compared with community or orthopedic control participants? J Neurotrauma 2017;34:2545-52.

51 Wilde EA, Ware AL, Li X, et al. Orthopedic injured versus uninjured comparison groups for neuroimaging research in mild traumatic brain injury. J Neurotrauma 2019;36:239-49. 
52 Osmond MH, Klassen TP, Wells GA, et al. Catch: a clinical decision rule for the use of computed tomography in children with minor head injury. Can Med Assoc J 2010;182:341-8.

53 Gagnon I, Friedman D, Beauchamp MH, et al. The Canadian pediatric mild traumatic brain injury common data elements project: Harmonizing outcomes to increase understanding of pediatric concussion. J Neurotrauma 2018;35:1849-57.

54 Beauchamp MH, Aglipay M, Yeates KO, et al. Predictors of neuropsychological outcome after pediatric concussion. Neuropsychology 2018;32:495-508.

55 Chang H-K, Hsu J-W, Wu J-C, et al. Traumatic brain injury in early childhood and risk of attention-deficit/hyperactivity disorder and autism spectrum disorder: a nationwide longitudinal study. J Clin Psychiatry 2018;79:pii: 17m11857.

56 Haarbauer-Krupa J, Lee $\mathrm{AH}$, Bitsko $\mathrm{RH}$, et al. Prevalence of ParentReported traumatic brain injury in children and associated health conditions. JAMA Pediatr 2018;172:1078-86.

57 von Elm E, Altman DG, Egger M, et al. The strengthening the reporting of observational studies in epidemiology (STROBE) statement: guidelines for reporting observational studies. J Clin Epidemiol 2008;61:344-9.

58 Laurendeau-Martin J, Beaudoin C, Désiré N, et al. Preliminary validation of the reactions questionnaire: a observational Postconcussive checklist for early childhood. J Int Neuropsychol Soc 2017;25:i-422.

59 Babl FE, Crellin D, Cheng J, et al. The use of the faces, legs, activity, Cry and Consolability scale to assess procedural pain and distress in young children. Pediatr Emerg Care 2012;28:1281-96.

60 Crellin DJ, Harrison D, Santamaria N, et al. Systematic review of the face, legs, activity, Cry and Consolability scale for assessing pain in infants and children. Pain 2015;156:2132-51.

61 Brigance A, French B. Brigance Inventory of Early Development III. Massachussets: Curriculum Associates, 2013.

62 Brigance AH, French B. Brigance Early Childhood Screens III. Massachussets: Curriculum Associates, 2013.

63 Korkman M, Kirk U, Kemp S. NEPSY-II: a developmental neuropsychological assessment. 2nd ed. San Antonio, Texas: The Psychological Corporation, 2007.

64 Psychometrics of the Minnesota Executive Function Scale. In paper symposium: strengthening the measurement toolbox: development and validation of technology-based executive function and cognitive assessments. Austin, Texas: Society for Research in Child Development, 2017.

65 Kochanska G, Murray K, Jacques TY, et al. Inhibitory control in young children and its role in emerging internalization. Child Dev 1996;67:490-507.

66 Reed MA, Pien DL, Rothbart MK. Inhibitory self-control in preschool. Merrill-Palmer Quarterly 1984;30:131-47.

67 Diamond A, Goldman-Rakic PS. Comparison of human infants and rhesus monkeys on Piaget's AB task: evidence for dependence on dorsolateral prefrontal cortex. Exp Brain Res 1989;74:24-40.

68 Ewing-Cobbs L, Prasad MR, Landry SH, et al. Executive functions following traumatic brain injury in young children: a preliminary analysis. Dev Neuropsychol 2004;26:487-512.

69 Warschausky S. Wechsler Preschool and Primary Scale of Intelligence. In: Kreutzer JS, DeLuca J, Caplan B, eds. Encyclopedia of Clinical Neuropsychology. New York, NY: Springer, 2011. https:// doi.org/10.1007/978-0-387-79948-3_1606

70 Mundy P, Delgado C, Block J, et al. A manual for the abridged Early Social Communication Scales (ESCS). Coral Gables, Florida: University of Miami, 2003.

71 Hutman T, Rozga A, DeLaurentis AD, et al. Response to distress in infants at risk for autism: a prospective longitudinal study. Journal of Child Psychology and Psychiatry 2010;51:1010-20.

72 Hutman T, Rozga A, DeLaurentis A, et al. Infants' pre-empathic behaviors are associated with language skills. Infant Behav Dev 2012;35:561-9.

73 Wiefferink CH, Rieffe C, Ketelaar L, et al. Emotion understanding in deaf children with a cochlear implant. $J$ Deaf Stud Deaf Educ 2013;18:175-86.

74 Gagne JR, Van Hulle CA, Aksan N, et al. Deriving childhood temperament measures from emotion-eliciting behavioral episodes: scale construction and initial validation. Psychol Assess 2011;23:337-53.

75 Aksan N, Kochanska G, Ortmann MR. Mutually responsive orientation between parents and their young children: toward methodological advances in the science of relationships. Dev Psychol 2006;42:833-48.

76 Kochanska G, Aksan N, Prisco TR, et al. Mother-child and fatherchild mutually responsive orientation in the first 2 years and children's outcomes at preschool age: mechanisms of influence. Child Dev 2008;79:30-44.

77 Bélanger Marie-Ève, Bernier A, Paquet J, et al. Validating actigraphy as a measure of sleep for preschool children. J Clin Sleep Med 2013;9:701-6.

78 Tétreault Émilie, Bernier A, Matte-Gagné C, et al. Normative developmental trajectories of actigraphic sleep variables during the preschool period: a three-wave longitudinal study. Dev Psychobiol 2019;61:141-53.

79 Frayne A, Benischek A, Walton W, et al. Factors associated with successful MRI scanning in unsedated young children. Front Pediatr 2018;6:146.

80 Tuerk C, Lacombe-Barrios J, Dégeilh F, et al. Who will make it through? Predicting non-sedated MRO succes in young children with and without traumatic brain injury. Brain Inj 2019;33:232.

81 Lee DY, Kim E, Choi MH. Technical and clinical aspects of cortisol as a biochemical marker of chronic stress. BMB Rep 2015;48:209-16.

82 Treble-Barna A, Pilipenko V, Wade SL, et al. Cumulative influence of inflammatory response genetic variation on long-term neurobehavioral outcomes after pediatric traumatic brain injury relative to orthopedic injury: an exploratory polygenic risk score. $J$ Neurotrauma 2020;37:1491-503.

83 Beers SR, Wisniewski SR, Garcia-Filion P, et al. Validity of a pediatric version of the Glasgow outcome Scale-Extended. $J$ Neurotrauma 2012;29:1126-39.

84 Briggs-Gowan MJ, Carter AS. Brief infant toddler social emotional assessment (BITSEA). Pearson 2006:17-19.

85 Briggs-Gowan MJ, Carter AS. Applying the Infant-Toddler Social \& Emotional Assessment (ITSEA) and Brief-ITSEA in early intervention. Infant Ment Health J 2007;28:564-83.

86 Goodman R. The strengths and difficulties questionnaire: a research note. J Child Psychol Psychiatry 1997;38:581-6.

87 Goodman R. Psychometric properties of the strengths and difficulties questionnaire. J Am Acad Child Adolesc Psychiatry 2001;40:1337-45.

88 Putnam SP, Helbig AL, Gartstein MA, et al. Development and assessment of short and very short forms of the infant behavior questionnaire-revised. J Pers Assess 2014;96:445-58.

89 Putnam SP, Rothbart MK. Development of short and very short forms of the children's behavior questionnaire. J Pers Assess 2006;87:102-12.

90 LeBuffe PA, Naglieri JA. Devereux early childhood assessment for preschoolers. Second edition. Lewisville, NC: Kaplan Early Learning Company, 2012.

91 Mackrain M, LeBuffe P, Powell G. Devereux early childhood assessment for infants and toddlers. Lewisville, NC: Kaplan Early Learning Company, 2007.

92 Harrison PL, Oakland T. Adaptive Behavior Assessment System - Second Edition. 3rd ed. Torrance, CA: Western Psychological Services, 2015.

93 Rieffe C, Ketelaar L, Wiefferink CH. Assessing empathy in young children: construction and validation of an empathy questionnaire (EmQue). Pers Individ Dif 2010;49:362-7.

94 Tahiroglu D, Moses LJ, Carlson SM, et al. The children's social understanding scale: construction and validation of a parent-report measure for assessing individual differences in children's theories of mind. Dev Psychol 2014;50:2485-97.

95 Varni JW, Burwinkle TM, Szer IS. The PedsQL multidimensional fatigue scale in pediatric rheumatology: reliability and validity. $J$ Rheumatol 2004;31:2494-500

96 Bruni O, Ottaviano S, Guidetti V, et al. The sleep disturbance scale for children (SDSC) construct ion and validation of an instrument to evaluate sleep disturbances in childhood and adolescence. $J$ Sleep Res 1996;5:251-61.

97 Achenbach TM, Rescorla LA. Manual for the ASEBA School-Age Forms \& Profiles. Burlington, VT: University of Vermont, Research Center for Children, Youth, \& Families, 2001.

98 Moriarty DG, Zack MM, Kobau R. The Centers for Disease Control and Prevention's Healthy Days Measures - population tracking of perceived physical and mental health over time. Health Qual Life Outcomes 2003;1:37-8.

99 Varni JW, Seid M, Kurtin PS. PedsQL 4.0: reliability and validity of the pediatric quality of life inventory version 4.0 generic core scales in healthy and patient populations. Med Care 2001:39:800-12.

100 Sabourin S, Valois P, Lussier Y. Development and validation of a brief version of the dyadic adjustment scale with a nonparametric item analysis model. Psychol Assess 2005;17:15-27.

101 Spanier GB. Dyadic Adjustment Scale (DAS). J Marriage Fam 1976;38:15-28. 
102 Epstein NB, Baldwin LM, Bishop DS. The McMaster family assessment device. J Marital Fam Ther 1983;9:171-80.

103 Zigmond AS, Snaith RP. The hospital anxiety and depression scale. Acta Psychiatr Scand 1983;67:361-70.

104 Lalonde G, Bernier A, Beaudoin C, et al. Factors contributing to parent-child interaction quality following mild traumatic brain injury in early childhood. J Neuropsychol 2020;14:1-22.

105 Geyer CJ. Practical Markov chain Monte Carlo. Stat Sci 1992;7:473-83.

106 Enders CK. Applied missing data analysis. New York, NY: Guilford Press, 2010.

107 Graham JW. Missing data analysis: making it work in the real world. Annu Rev Psychol 2009;60:549-76.

108 Royo JL, Hidalgo M, Ruiz A. Pyrosequencing protocol using a universal biotinylated primer for mutation detection and SNP genotyping. Nat Protoc 2007;2:1734-9.

109 Meltzer LJ, Walsh CM, Traylor J, et al. Direct comparison of two new actigraphs and polysomnography in children and adolescents. Sleep 2012;35:159-66.

110 Hsieh H-F, Shannon SE. Three approaches to qualitative content analysis. Qual Health Res 2005;15:1277-88.
111 Catroppa C, Hearps S, Crossley L, et al. Social and behavioral outcomes following childhood traumatic brain injury: what predicts outcome at 12 months post-insult? J Neurotrauma 2017:34:1439-47.

112 Novak Z, Aglipay M, Barrowman N, et al. Association of persistent Postconcussion symptoms with pediatric quality of life. JAMA Pediatr 2016;170:e162900.

113 Plourde V, Yeates KO, Brooks BL. Predictors of long-term psychosocial functioning and health-related quality of life in children and adolescents with prior concussions. J Int Neuropsychol Soc 2018:24:540-8.

114 Kutcher JS, Giza CC. Sports concussion diagnosis and management. Continuum 2014;20:1552-69.

115 Holland SK, Altaye M, Robertson S, et al. Data on the safety of repeated $\mathrm{MRI}$ in healthy children. Neuroimage Clin 2014;4:526-30.

116 Connell A, Bullock BM, Dishion TJ, et al. Family intervention effects on co-occurring early childhood behavioral and emotional problems: a latent transition analysis approach. J Abnorm Child Psychol 2008;36:1211-25. 